YITP-18-05

\title{
Merlin-Arthur with efficient quantum Merlin and quantum supremacy for the second level of the Fourier hierarchy
}

\author{
Tomoyuki Morimae, ${ }^{1,2,3, *}$ Yuki Takeuchi, $, 4,5$, + and Harumichi Nishimura ${ }^{6, t}$ \\ ${ }^{1}$ Yukawa Institute for Theoretical Physics, Kyoto University, \\ Kitashirakawa Oiwakecho, Sakyo-ku, Kyoto 606-8502, Japan \\ ${ }^{2}$ Department of Computer Science, Gunma University, \\ 1-5-1 Tenjincho, Kiryu, Gunma, 376-0052, Japan \\ ${ }^{3}$ JST, PRESTO, 4-1-8 Honcho, Kawaguchi, Saitama, 332-0012, Japan \\ ${ }^{4}$ Graduate School of Engineering Science, \\ Osaka University, Toyonaka, Osaka 560-8531, Japan \\ ${ }^{5}$ NTT Communication Science Laboratories, \\ NTT Corporation, 3-1 Morinosato Wakamiya, \\ Atsugi, Kanagawa 243-0198, Japan \\ ${ }^{6}$ Graduate School of Informatics, Nagoya University, \\ Furocho, Chikusaku, Nagoya, Aichi, 464-8601, Japan
}

(Dated: November 13, 2018) 


\begin{abstract}
We introduce a simple sub-universal quantum computing model, which we call the Hadamardclassical circuit with one-qubit (HC1Q) model. It consists of a classical reversible circuit sandwiched by two layers of Hadamard gates, and therefore it is in the second level of the Fourier hierarchy [1]. We show that output probability distributions of the HC1Q model cannot be classically efficiently sampled within a multiplicative error unless the polynomial-time hierarchy collapses to the second level. The proof technique is different from those used for previous sub-universal models, such as IQP, Boson Sampling, and DQC1, and therefore the technique itself might be useful for finding other sub-universal models that are hard to classically simulate. We also study the classical verification of quantum computing in the second level of the Fourier hierarchy. To this end, we define a promise problem, which we call the probability distribution distinguishability with maximum norm (PDDMax). It is a promise problem to decide whether output probability distributions of two quantum circuits are far apart or close. We show that PDD-Max is BQP-complete, but if the two circuits are restricted to some types in the second level of the Fourier hierarchy, such as the HC1Q model or the IQP model, PDD-Max has a Merlin-Arthur system with quantum polynomial-time Merlin and classical probabilistic polynomial-time Arthur.
\end{abstract}

\footnotetext{
*Electronic address: tomoyuki.morimae@yukawa.kyoto-u.ac.jp

${ }^{\dagger}$ Electronic address: takeuchi.yuki@lab.ntt.co.jp

${ }^{\ddagger}$ Electronic address: hnishimura@is.nagoya-u.ac.jp
} 


\section{INTRODUCTION}

\section{A. Quantum supremacy of the HC1Q model}

The Fourier hierarchy [1] is a hierarchy of restricted quantum circuits. The $k$ th level of the Fourier hierarchy, $\mathrm{FH}_{k}$, is the class of quantum circuits with $k$ layers of Hadamard gates and all other gates preserving the computational basis. The second level, $\mathrm{FH}_{2}$, is the most important, because circuits in $\mathrm{FH}_{2}$ are used in many algorithms, such as Simon's algorithm [2] and Shor's factoring algorithm [3]. The instantaneous quantum polynomialtime (IQP) model [4, 5] is also in $\mathrm{FH}_{2}$ :

Definition 1 (IQP) The IQP model on $N$ qubits is the following quantum computing model:

1. The initial state is $\left|0^{N}\right\rangle$.

2. The unitary $H^{\otimes N} D H^{\otimes N}$ is applied. Here $H$ is the Hadamard gate, and $D$ is a quantum circuit consisting of only $Z$-diagonal gates, such as $Z, C Z, C C Z$, and $e^{i \theta Z}$, etc.

3. All qubits are measured in the computational basis.

The IQP model is a well-known example of sub-universal quantum computing models whose output probability distributions cannot be classically efficiently sampled unless the polynomial-time hierarchy collapses. Since a collapse of the polynomial-time hierarchy is considered as highly unlikely in computer science, it shows the hardness of classically simulating the IQP model. Other sub-universal models that exhibit similar quantum supremacy are also known, such as the depth four model [6], the Boson sampling model [7], the DQC1 model [8 12], the Fourier sampling model [13], the conjugated Clifford model [14], and the random circuit model [15].

The first main result of the present paper is to add another simple model in $\mathrm{FH}_{2}$ to the above list of sub-universal models that exhibit quantum supremacy. We define the Hadamard-classical circuit with one-qubit (HC1Q) model as follows:

Definition 2 (HC1Q) The Hadamard-classical circuit with one-qubit (HC1Q) model on $N$ qubits is the following quantum computing model (see Fig. 1(a)): 
1. The initial state is $\left|0^{N}\right\rangle$.

2. $H^{\otimes(N-1)} \otimes I$ is applied.

3. A polynomial-time uniformly-generated classical reversible circuit

$$
C:\{0,1\}^{N} \ni w \mapsto C(w) \in\{0,1\}^{N}
$$

is applied "coherently".

4. $H^{\otimes(N-1)} \otimes I$ is applied.

5. All qubits are measured in the computational basis.

(a)

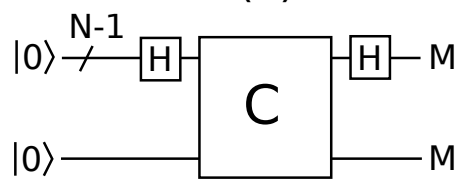

(b)

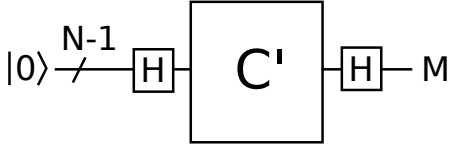

(c)

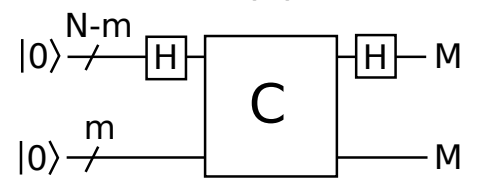

FIG. 1: (a) The HC1Q model. $M$ is the computational-basis measurement. A single line with the slash represents a set of $N-1$ qubits. The Hadamard gate $H$ is applied on each qubit. The measurement $M$ is done on each qubit. (b) The circuit similar to HC1Q model, but the last $|0\rangle$ qubit is removed. (c) The generalization of the $\mathrm{HC1Q}$ model, which we call the $\mathrm{HC}$ Q model.

We show that the HC1Q model is universal with a postselection. More precisely, we show the following:

Theorem 3 Let $U$ be a polynomial-time uniformly-generated quantum circuit acting on $n$ qubits that consists of poly $(n)$ number of Hadamard gates and classical reversible gates, such as $X$, CNOT, and Toffoli. Then from $U$ we can efficiently construct an HC1Q circuit on $N=\operatorname{poly}(n)$ qubits such that a postselection on some output qubits generates the state $U\left|0^{n}\right\rangle$. 
Since Hadamard plus classical gates are universal, Theorem 3 shows that HC1Q model is universal with a postselection. A proof of the theorem is given in Sec. II. The proof is based on a new idea that is different from those used in the previous results [4, 6, 7, 9]. Proofs for the depth-four model [6], the Boson sampling model [7], and the IQP model [4] use gadgets that insert gates in a post hoc way by using postselections. (For example, the IQP model is not universal because Hadamard gates can be applied only in the first layer and the last layer, but the so-called Hadamard gadget [4] enables realizations of Hadamard gates in any place of the IQP model by using postselections.) The proof for the DQC1 model [9] uses a postselection to initialize the maximally-mixed state $I^{\otimes n} / 2^{n}$ to the pure state $\left|0^{n}\right\rangle$. As is explained in Sec. II, our proof is different from those: from $U$, we first construct a polynomial-time non-deterministic algorithm. From the polynomial-time nondeterministic algorithm, we next construct a classical deterministic polynomial-time circuit $C$. We finally show that the $\mathrm{HC} 1 \mathrm{Q}$ circuit $\left(H^{\otimes N-1} \otimes I\right) C\left(H^{\otimes N-1} \otimes I\right)$ can generate $U\left|0^{n}\right\rangle$ with a postselection. This idea itself seems to be useful for finding other new sub-universal models that exhibit quantum supremacy. We do not know relations between our technique and previous ones.

By using the arguments of Ref. [4, 7, 9], we obtain the following corollary of Theorem 3 , which is quantum supremacy of the HC1Q model:

Corollary 4 Output probability distributions of the HC1Q model cannot be classically efficiently sampled within a multiplicative error $\epsilon<1$ unless the polynomial-time hierarchy collapses to the third level.

Here, we say that a probability distribution $\left\{p_{z}\right\}_{z}$ is classically efficiently sampled within a multiplicative error $\epsilon$ if there exists a classical probabilistic polynomial-time algorithm such that

$$
\left|p_{z}-q_{z}\right| \leq \epsilon p_{z}
$$

for all $z$, where $q_{z}$ is the probability that the algorithm outputs $z$.

The corollary demonstrates an interesting "phase transition" between classical and quantum. To see it, let us consider the circuit of Fig. 1(b), which is obtained by removing the last $|0\rangle$ qubit of the HC1Q model (Fig. 1(a)). Here,

$$
C^{\prime}:\{0,1\}^{N-1} \ni w \mapsto C^{\prime}(w) \in\{0,1\}^{N-1}
$$


is a polynomial-time uniformly-generated classical reversible circuit. The circuit of Fig. 1(b) is trivially classically simulatable, since $C^{\prime}$ is a permutation on $\{0,1\}^{N-1}$ and therefore

$$
C^{\prime}|+\rangle^{\otimes(N-1)}=\frac{1}{\sqrt{2^{N-1}}} \sum_{x \in\{0,1\}^{N-1}}\left|C^{\prime}(x)\right\rangle=|+\rangle^{\otimes(N-1)}
$$

where $|+\rangle \equiv(|0\rangle+|1\rangle) / \sqrt{2}$. Our result therefore suggests that the addition of a single $|0\rangle$ qubit to the trivial circuit of Fig. 1(b) changes its complexity dramatically.

The third level collapse of the polynomial-time hierarchy for the depth-four model, the Boson sampling model, the IQP model, and the DQC1 model can be improved to the second level collapse [11, 12]. In Sec. III, we show that the same improvement is possible for the HC1Q model:

Theorem 5 Output probability distributions of the HC1Q model cannot be classically efficiently sampled within a multiplicative error $\epsilon<1$ unless the polynomial-time hierarchy collapses to the second level.

A natural question is whether the above supremacy results still hold when the number of qubits measured is much reduced. It is known that for the $N$-qubit IQP model probability distributions of measurement results on $O(\log (N))$ number of qubits can be classically exactly sampled in polynomial time [4]. In Sec. IV] we show an analogous result for the $\mathrm{HC1Q}$ model:

Theorem 6 Probability distributions of measurement results on $O(\log (N))$ number of qubits in the HC1Q model can be classically sampled in polynomial time within a $1 / p o l y(N)$ L1-norm error.

It is possible to extend this result to a generalized version of the $\mathrm{HC} 1 \mathrm{Q}$ model, which we call the $\mathrm{HC} m \mathrm{Q}$ model:

Definition 7 ( $\mathbf{H C m} \mathbf{Q})$ The Hadamard-classical circuit with $m$-qubit ( $\mathrm{HCmQ}$ ) model on $N$ qubits is the following quantum computing model (see Fig. 1(c)):

1. The initial state is $\left|0^{N}\right\rangle$.

2. $H^{\otimes(N-m)} \otimes I^{\otimes m}$ is applied. 
3. A polynomial-time uniformly-generated classical reversible circuit

$$
C:\{0,1\}^{N} \ni w \mapsto C(w) \in\{0,1\}^{N}
$$

is applied "coherently".

4. $H^{\otimes(N-m)} \otimes I^{\otimes m}$ is applied.

5. All qubits are measured in the computational basis.

Theorem 8 Probability distributions of measurement results on $O(\log (N))$ number of

qubits in the $\mathrm{HCm}$ Q model can be classically sampled in polynomial time within a $1 / \operatorname{poly}(N)$ L1-norm error.

Its proof is omitted since it is similar to that of Theorem 6.

At this moment, we do not know which sub-universal model is the most promising for experimental realizations, but the $\mathrm{HC} 1 \mathrm{Q}$ model should be useful for certain experimental setups due to its simple structure.

\section{B. The verification of quantum computing in the second level of the Fourier hi- erarchy}

In this paper, we also study the classical verification of quantum computing. It is a longstanding open problem whether quantum computing is classically verifiable. More precisely, it is open whether any problem $L$ in BQP has an interactive proof system with a quantum polynomial-time prover and a classical probabilistic polynomial-time verifier.

Definition 9 We say that a problem $L$ has an interactive proof system with a quantum polynomial-time prover if there exists a classical probabilistic polynomial-time verifier such that

- If $x \in L$ then there exists a quantum polynomial-time prover such that the verifier accepts with probability at least $2 / 3$.

- If $x \notin L$ then for any prover the verifier accepts with probability at most $1 / 3$. 
(Note that in this definition, the prover is in quantum polynomial-time only for yes instances, i.e., when the prover is honest. For no instances, the computational power of the malicious prover is unbounded.)

Answering the open question is important not only for practical applications of cloud quantum computing but also for foundations of computer science and quantum physics [16]. In fact, several partial solutions to the open problem have been obtained. They are categorized into the following four types:

1. Several verification protocols [17, 18] and verifiable blind quantum computing protocols [19-23] demonstrate that if the verifier has a weak quantum ability, such as preparations or measurements of single-qubit quantum states, any BQP problem can be verified with a quantum polynomial-time prover.

2. If multiple entangling quantum polynomial-time provers who are not communicating with each other are allowed, any BQP problem is verified with a classical polynomialtime verifier [24-26].

3. Since BQP is contained in IP [27], a natural approach to the open problem is to restrict the prover of IP to quantum polynomial-time when the problem is in BQP. In fact, recently, a step in this line has been obtained in Ref. [28]. The authors of Ref. [28] have constructed a new interactive proof system that verifies the value of the trace of operators with a postBQP prover and a classical polynomial-time verifier.

4. It has been shown recently that the classical verification of quantum computing is possible if a certain problem is assumed to be hard for quantum computing [29].

Actually, the answer to the open problem is unconditionally yes if we consider specific BQP problems. For example, it is known that the recursive Fourier sampling [30] has an interactive proof system with a quantum polynomial-time prover and a classical polynomialtime verifier who communicate polynomial number of messages [31]. Furthermore, it has been shown recently that calculating orders of solvable groups has an interactive proof system with a quantum polynomial-time prover and a classical polynomial-time verifier who exchange two or three messages [33]. Finally, it was suggested in Ref. [32] that a problem of deciding whether there exist some results that occur with high probability or not for circuits in $\mathrm{FH}_{2}$ has a Merlin-Arthur system with quantum polynomial-time Merlin. 
Definition 10 We say that a problem $L$ has a Merlin-Arthur system with a quantum polynomial-time Merlin if $L$ has an interactive proof system with a quantum polynomialtime prover (Merlin) and a classical probabilistic polynomial-time verifier (Arthur) where only a single message transmission is done from the prover to the verifier.

The second main result of the present paper is to introduce another problem in BQP that is classically verifiable. More precisely, we define the following promise problem that we call Probability Distribution Distinguishability with Maximum Norm (PDD-Max):

Definition 11 (PDD-Max) Given a classical description of two quantum circuits $U_{1}$ and $U_{2}$ acting on $N$ qubits that consist of poly $(N)$ number of elementary gates, and parameters $a$ and $b$ such that $0 \leq b<a \leq 1$ and $a-b \geq 1 / \operatorname{poly}(N)$, decide

- YES: there exists $z \in\{0,1\}^{N}$ such that $\left|p_{z}-q_{z}\right| \geq a$.

- NO: for any $z \in\{0,1\}^{N},\left|p_{z}-q_{z}\right| \leq b$.

Here, $p_{z} \equiv\left|\left\langle z\left|U_{1}\right| 0^{N}\right\rangle\right|^{2}$ and $q_{z} \equiv\left|\left\langle z\left|U_{2}\right| 0^{N}\right\rangle\right|^{2}$.

We first show that PDD-Max exactly characterizes the power of BQP:

Theorem 12 PDD-Max is BQP-complete (under polynomial-time many-one reduction).

Its proof is given in Sec. V. (Note that if two circuits are classical, PDD-Max is BPPcomplete; we have only to replace the controlled- $H$ gate in Fig. 3 with a controlled-randombit-flip gate.)

We next show that if $U_{1}$ and $U_{2}$ of PDD-Max are restricted to the HC1Q model, PDD-Max is classically verifiable:

Theorem 13 If two circuits $U_{1}$ and $U_{2}$ are in the form of the HC1Q model, PDD-Max has a Merlin-Arthur system with quantum polynomial-time Merlin.

The proof of Theorem 13 is given in Sec. VI. A similar proof holds for the IQP model and the $\mathrm{HC} m \mathrm{Q}$ model (including the Simon-type circuits) (see Sec. VIIA).

These results demonstrate that if we restrict the circuits of PDD-Max to the form of the HC1Q model, it is another example of problems that is classically verifiable. These results also suggest that such a restriction of PDD-Max is not BQP-hard, since BQP is not believed to be in MA [34]. 
The classical verifiability of PDD-Max for restricted circuits does not seem to be directly related to the classical verification of quantum supremacy, such as the verification of the IQP model, but it is an important future research subject to explore any relation between them.

\section{Preliminary}

In this paper, we often use the following well known inequality:

Theorem 14 (Chernoff-Hoeffding bound) Let $X_{1}, \ldots, X_{T}$ be identically and independently distributed real random variables with $\left|X_{i}\right| \leq 1$ for every $i=1, \ldots, T$. Then

$$
\operatorname{Pr}\left[\left|\frac{1}{T} \sum_{i=1}^{T} X_{i}-E\left(X_{i}\right)\right| \leq \epsilon\right] \geq 1-2 e^{-\frac{T \epsilon^{2}}{2}} .
$$

In the following sections, we give proofs of theorems. In the last section, Sec. VII, we provide some discussions.

\section{PROOF OF THEOREM 3}

In this section, we show Theorem 3. We are given a unitary operator

$$
U=u_{t} \ldots u_{2} u_{1}
$$

acting on $n$ qubits, where $u_{i}$ is the Hadamard gate $H$ or a classical gate for all $i=1,2, \ldots, t$. From $U$, we define

$$
U^{\prime} \equiv u_{t+n} \ldots u_{t+1} U
$$

where $u_{t+i}$ is $H$ acting on $i$ th qubit $(i=1,2, \ldots, n)$.

The outline of our proof is as follows. We first construct a polynomial-time nondeterministic algorithm from $U^{\prime}$. We next define a polynomial-time quantum unitary operator $W$, which consists of only classical gates, from the polynomial-time non-deterministic algorithm. We finally show that the HC1Q model of Fig. 2 that uses $W$ is universal with a postselection.

Now let us consider the following polynomial-time non-deterministic algorithm. 


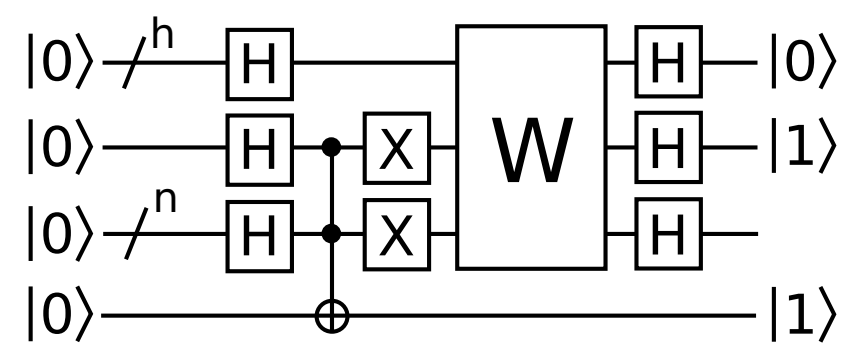

FIG. 2: The quantum circuit with a postselection. The first $h$ qubits, the $(h+1)$ th qubit, and the last qubit are postselected to $\left|0^{h}\right\rangle,|1\rangle$, and $|1\rangle$, respectively.

0 . The state of the register is represented by $(s, z)$, where $s \in\{0,1\}$ and $z \in\{0,1\}^{n}$.

1. The initial state of the register is $\left(s=0, z=0^{n}\right)$.

2. Repeat the following for $i=1, \ldots, t+n$.

2-a. If $u_{i}$ is a classical gate (such as $X$, CNOT, or Toffoli) whose corresponding action on $\{0,1\}^{n}$ is

$$
g:\{0,1\}^{n} \ni z \mapsto g(z) \in\{0,1\}^{n}
$$

update the state of the register as

$$
(s, z) \rightarrow(s, g(z))
$$

2-b. If $u_{i}$ is $H$ acting on $j$ th qubit, update the state of the register in the following non-deterministic way:

$$
(s, z) \rightarrow\left\{\begin{array}{l}
\left(s, z_{1}, \ldots, z_{j-1}, 0, z_{j+1}, \ldots, z_{n}\right) \\
\left(s \oplus z_{j}, z_{1}, \ldots, z_{j-1}, 1, z_{j+1}, \ldots, z_{n}\right) .
\end{array}\right.
$$

Let us define $h$ by

$$
h \equiv\left|\left\{i \in\{1,2, \ldots, t+n\} \mid u_{i}=H\right\}\right|,
$$

i.e., $h$ is the number of $H$ appearing in $U^{\prime}$. The above polynomial-time non-deterministic algorithm does the non-deterministic transition $h$ times, and therefore the algorithm has $2^{h}$ computational paths. We label each path by an $h$-bit string $y \in\{0,1\}^{h}$. We write the 
final state of the register for the path $y \in\{0,1\}^{h}$ by $(s(y), z(y))$, where $s(y) \in\{0,1\}$ and $z(y) \in\{0,1\}^{n}$. Then, we obtain

$$
U^{\prime}\left|0^{n}\right\rangle=\frac{1}{\sqrt{2^{h}}} \sum_{y \in\{0,1\}^{h}}(-1)^{s(y)}|z(y)\rangle .
$$

(To help the readers understand the derivation of Eq. (1), we provide a simple example in Appendix A.)

Note that, for each $y$, we can calculate $s(y)$ and $z(y)$ in classical polynomial-time. Let us define the polynomial-time unitary operator $W$ by

$$
W\left(|y\rangle \otimes|0\rangle \otimes\left|0^{n}\right\rangle\right)=|y\rangle \otimes|s(y)\rangle \otimes|z(y)\rangle
$$

It is easy to see that $W$ can be constructed with only classical gates. Now we show that the state $H^{\otimes n} U^{\prime}\left|0^{n}\right\rangle=U\left|0^{n}\right\rangle$ can be generated by the HC1Q model of Fig. 2 that uses $W$ with a postselection. In Fig. 2, the state immediately before the postselection is

$$
\frac{1}{\sqrt{2^{n+h+1}}} \sum_{y \in\{0,1\}^{h}}\left(H^{\otimes h}|y\rangle\right) \otimes(H|s(y)\rangle) \otimes\left(H^{\otimes n}|z(y)\rangle\right) \otimes|1\rangle+|\psi\rangle \otimes|0\rangle,
$$

where $|\psi\rangle$ is a certain $(h+n+1)$-qubit state whose detail is irrelevant [35]. After the postselection, the state becomes

$$
\frac{1}{\sqrt{2^{h}}} \sum_{y \in\{0,1\}^{h}}(-1)^{s(y)} H^{\otimes n}|z(y)\rangle=H^{\otimes n} U^{\prime}\left|0^{n}\right\rangle=U\left|0^{n}\right\rangle .
$$

Hence, we have shown that the HC1Q model of Fig. 2 with a postselection can generate $U\left|0^{n}\right\rangle$ for any unitary $U$ that consists of Hadamard and classical gates, which means that the $\mathrm{HC} 1 \mathrm{Q}$ model is universal with a postselection.

\section{PROOF OF THEOREM [5}

It was shown in Refs. [11, 12] that the third level collapse of the polynomial-time hierarchy for most of the sub-universal models (including the depth-four model [6], the Boson sampling model [7], the IQP model [4], and the DQC1 model [9]) is improved to the second level collapse. The idea is to use the class NQP [37] in stead of postBQP. NQP is a quantum version of NP, and defined as follows: 
Definition 15 (NQP) A promise problem $A=\left(A_{y e s}, A_{n o}\right)$ is in NQP if and only if there exists a polynomial-time uniformly-generated family $\left\{V_{x}\right\}_{x}$ of quantum circuits such that if $x \in A_{\text {yes }}$ then $p_{a c c}>0$, and if $x \in A_{n o}$ then $p_{a c c}=0$. Here $p_{a c c}$ is the acceptance probability of $V_{x}$.

If we remember the following definition of NP, it is clear that NQP is a quantum version of NP.

Definition 16 (NP) A promise problem $A=\left(A_{y e s}, A_{n o}\right)$ is in NP if and only if there exists a classical polynomial-time probabilistic algorithm such that if $x \in A_{\text {yes }}$ then $p_{\text {acc }}>0$, and if $x \in A_{n o}$ then $p_{a c c}=0$. Here $p_{a c c}$ is the acceptance probability of the classical algorithm.

By using a similar argument of Refs. [11, 12], we now show that the collapse of the polynomial-time hierarchy to the third level for the HC1Q model can be improved to the second level. Let $A=\left(A_{y e s}, A_{n o}\right)$ be a promise problem in NQP. Then there exists a polynomial-time uniformly-generated family $\left\{V_{x}\right\}_{x}$ of quantum circuits that satisfies the above definition of NQP. The outline of our proof is as follows: First, from $V_{x}$, we construct an HC1Q circuit. Second, we assume that its output probability distribution is classically efficiently sampled within a multiplicative error $\epsilon<1$. Finally, we show that then $A$ is in $\mathrm{NP}$, which means NQP $\subseteq$ NP. It collapses the polynomial-time hierarchy to the second level, since

$$
\mathrm{PH} \subseteq \mathrm{BP} \cdot \mathrm{coC}_{=} \mathrm{P}=\mathrm{BP} \cdot \mathrm{NQP}=\mathrm{BP} \cdot \mathrm{NP}=\mathrm{AM}
$$

Now let us give a more precise proof. In Sec. II], we have constructed the HC1Q circuit of Fig. 2 from a given unitary $U$. Let us repeat the same argument by replacing $U$ with $V_{x}$. In stead of doing the postselection on the state of Eq. (2), let us do the projective measurement $\left\{\Lambda, I^{\otimes h+n+2}-\Lambda\right\}$ on it, where

$$
\Lambda \equiv|0\rangle\left\langle\left. 0\right|^{\otimes h} \otimes \mid 1\right\rangle\langle 1|\otimes| 0\rangle\left\langle 0\left|\otimes I^{\otimes n-1} \otimes\right| 1\right\rangle\langle 1| .
$$

The probability $p_{\Lambda}$ of obtaining $\Lambda$ is

$$
p_{\Lambda}=\frac{\left\langle 0^{n}\left|V_{x}^{\dagger}\left(|0\rangle\langle 0| \otimes I^{\otimes n-1}\right) V_{x}\right| 0^{n}\right\rangle}{2^{h+n+2}} .
$$

By the definition of NQP,

$$
\left\langle 0^{n}\left|V_{x}^{\dagger}\left(|0\rangle\langle 0| \otimes I^{\otimes n-1}\right) V_{x}\right| 0^{n}\right\rangle>0
$$


when $x \in A_{\text {yes }}$ and

$$
\left\langle 0^{n}\left|V_{x}^{\dagger}\left(|0\rangle\langle 0| \otimes I^{\otimes n-1}\right) V_{x}\right| 0^{n}\right\rangle=0
$$

when $x \in A_{n o}$. It means that $p_{\Lambda}>0$ when $x \in A_{\text {yes }}$ and $p_{\Lambda}=0$ when $x \in A_{n o}$.

Assume that $p_{\Lambda}$ is classically efficiently sampled within a multiplicative error $\epsilon<1$. It means that there exists a classical polynomial-time probabilistic algorithm that outputs 0 or 1 such that

$$
\left|p_{\Lambda}-q_{0}\right| \leq \epsilon p_{\Lambda},
$$

where $q_{0}$ is the probability that the classical algorithm outputs 0 (accepts). Then, we can show that $A$ is in NP. In fact, if $x \in A_{\text {yes }}$ then

$$
q_{0} \geq(1-\epsilon) p_{\Lambda}>0 .
$$

If $x \in A_{n o}$ then

$$
q_{0} \leq(1+\epsilon) p_{\Lambda}=0 .
$$

According to Definition [16, $A$ is therefore in NP.

\section{PROOF OF THEOREM 6}

In this section, we show Theorem 6. For simplicity, we assume that the first $k$ qubits are measured, where $k=O(\log N)$. Generalizations to other $k$ qubits are the same.

The outline of our proof is as follows. We first construct a probability distribution $\left\{q_{z}\right\}_{z}$ that can be calculated in classical polynomial time, and is close to $\left\{p_{z}\right\}_{z}$ within a $1 /$ poly L1-norm. We then show that we can sample $\left\{q_{z}\right\}_{z}$ in classical polynomial time.

Let us consider the circuit of Fig. 1 (a). As is shown in Appendix B, the probability of obtaining the measurement result $z \in\{0,1\}^{k}$ for the first $k$ qubits is

$$
p_{z}=\frac{1}{2^{N-1}} \sum_{x \in\{0,1\}^{N-1}} f(x),
$$

where

$$
\begin{aligned}
f(x) & \equiv \frac{1}{2^{k}} \sum_{y \in\{0,1\}^{N-1}}(-1)^{z \cdot C_{1, \ldots, k}(x 0)+z \cdot C_{1, \ldots, k}(y 0)} \delta_{C_{k+1, \ldots, N}(x 0), C_{k+1, \ldots, N}(y 0)} \\
& =\frac{1}{2^{k}} \sum_{y \in S}(-1)^{z \cdot C_{1, \ldots, k}(x 0)+z \cdot C_{1, \ldots, k}(y 0)}
\end{aligned}
$$


$C_{1, \ldots, k}(x 0)$ is the first $k$ bits of $C(x 0), C_{k+1, \ldots, N}(x 0)$ is the last $N-k$ bits of $C(x 0)$, and $S \subseteq\{0,1\}^{N-1}$ is defined by

$$
S \equiv\left\{y \in\{0,1\}^{N-1} \mid C_{k+1, \ldots, N}(x 0)=C_{k+1, \ldots, N}(y 0)\right\} .
$$

The subset $S$ can be obtained in polynomial time in the following way:

1. Set $S=\{\}$.

2. Repeat the following for all $\alpha \in\{0,1\}^{k}$.

2-1. Calculate $C^{-1}\left(\alpha C_{k+1, \ldots, N}(x 0)\right)$.

2-2. If $C^{-1}\left(\alpha C_{k+1, \ldots, N}(x 0)\right)=y 0$ for certain $y \in\{0,1\}^{N-1}$, add $y$ to $S$.

3. End.

From the construction, $|S| \leq 2^{k}=$ poly $(N)$. Therefore, the value of $f(x)$ is exactly computable in polynomial time for each $x$. Furthermore, $|f(x)| \leq 1$ because

$$
|f(x)| \leq \frac{1}{2^{k}} \sum_{y \in S}\left|(-1)^{z \cdot C_{1, \ldots, k}(x 0)+z \cdot C_{1, \ldots, k}(y 0)}\right| \leq 1 .
$$

Let us generate random bit strings $x_{1}, \ldots, x_{T} \in\{0,1\}^{N-1}$. We then calculate

$$
\tilde{p}_{z} \equiv \frac{1}{T} \sum_{i=1}^{T} f\left(x_{i}\right)
$$

Note that if we take $X_{i}=f\left(x_{i}\right)$, then $E\left(X_{i}\right)=p_{z}$, and therefore from the Chernoff-Hoeffding bound,

$$
\operatorname{Pr}\left[\left|\tilde{p}_{z}-p_{z}\right| \leq \epsilon\right] \geq 1-2 e^{-\frac{T \epsilon^{2}}{2}} .
$$

For any polynomial $r$, let us take $\epsilon=\frac{1}{5 \times 2^{2 k_{r}}}$. Given $\left\{\tilde{p}_{z}\right\}_{z}$, define the probability distribution $\left\{q_{z}\right\}_{z}$ by

$$
q_{z} \equiv \frac{\left|\tilde{p}_{z}\right|}{\sum_{z \in\{0,1\}^{k}}\left|\tilde{p}_{z}\right|}
$$

Note that it is well defined, because

$$
\sum_{z \in\{0,1\}^{k}}\left|\tilde{p}_{z}\right|>0
$$


which is shown as follows:

$$
\sum_{z \in\{0,1\}^{k}}\left|\tilde{p}_{z}\right| \geq \sum_{z \in\{0,1\}^{k}} \tilde{p}_{z} \geq \sum_{z \in\{0,1\}^{k}}\left(p_{z}-\epsilon\right)=1-2^{k} \epsilon=1-\frac{1}{5 \times 2^{k} r}>0 .
$$

Furthermore, $\left\{q_{z}\right\}_{z}$ is obtained in polynomial time. The distance between $\left\{p_{z}\right\}_{z}$ and $\left\{q_{z}\right\}_{z}$ is

$$
\sum_{z \in\{0,1\}^{k}}\left|p_{z}-q_{z}\right| \leq 5 \times 2^{2 k} \epsilon=\frac{1}{r}
$$

It is shown as follows. First,

$$
\begin{aligned}
q_{z} & =\frac{\left|\tilde{p}_{z}\right|}{\sum_{z \in\{0,1\}^{k}}\left|\tilde{p}_{z}\right|} \\
& \leq \frac{p_{z}+\epsilon}{1-2^{k} \epsilon} \\
& =\left(p_{z}+\epsilon\right)\left(1+2^{k} \epsilon+o\left(2^{k} \epsilon\right)\right) \\
& =p_{z}+p_{z} 2^{k} \epsilon+p_{z} O\left(2^{k} \epsilon\right)+\epsilon+2^{k} \epsilon^{2}+\epsilon o\left(2^{k} \epsilon\right) \\
& \leq p_{z}+5 \times 2^{k} \epsilon .
\end{aligned}
$$

Second,

$$
\begin{aligned}
q_{z} & =\frac{\left|\tilde{p}_{z}\right|}{\sum_{z \in\{0,1\}^{k}}\left|\tilde{p}_{z}\right|} \\
& \geq \frac{p_{z}-\epsilon}{1+2^{k} \epsilon} \\
& =\left(p_{z}-\epsilon\right)\left(1-2^{k} \epsilon+o\left(2^{k} \epsilon\right)\right) \\
& =p_{z}-p_{z} 2^{k} \epsilon+p_{z} o\left(2^{k} \epsilon\right)-\epsilon+2^{k} \epsilon^{2}-\epsilon o\left(2^{k} \epsilon\right) \\
& \geq p_{z}-5 \times 2^{k} \epsilon .
\end{aligned}
$$

In this way, we have shown that we can calculate in classical polynomial time the probability distribution $\left\{q_{z}\right\}_{z}$ that is close to $\left\{p_{z}\right\}_{z}$. Our final task is to show that $\left\{q_{z}\right\}_{z}$ can be sampled in classical polynomial time. For simplicity, let us assume that each $q_{z}$ is represented in the $m$-bit binary:

$$
q_{z}=\sum_{j=1}^{m} 2^{-j} a_{z, j}
$$

where $a_{z, j} \in\{0,1\}$ for $j=1,2, \ldots, m$. (Otherwise, by polynomially increasing the size of $m$, we obtain exponentially good approximations.) The following algorithm samples the probability distribution $\left\{q_{z}\right\}_{z}$. 
1. Randomly generate an $m$-bit string $\left(w_{1}, \ldots, w_{m}\right) \in\{0,1\}^{m}$.

2. Output $z \in\{0,1\}^{k}$ such that

$$
\sum_{y<z} q_{y} \leq \sum_{j=1}^{m} 2^{-j} w_{j}<\sum_{y \leq z} q_{y}
$$

where $y<z$ and $y \leq z$ mean the standard dictionary order. (For example, for three bits, $000<001<010<011<100<101<110<111$.)

In summary, we have shown that $\left\{p_{z}\right\}_{z}$ can be sampled in polynomial time within a 1/poly $(N)$ L1-norm error.

\section{PROOF OF THEOREM 12}

In this section, we show Theorem 12, Our proof consists of two parts. In the first subsection, we show that PDD-Max is BQP-hard. In the second subsection, we show that PDD-Max is in BQP.

\section{A. BQP-hardness}

Let $A=\left(A_{\text {yes }}, A_{n o}\right)$ be a promise problem in BQP. It means that there exists a uniform family $\left\{V_{x}\right\}_{x}$ of polynomial-size quantum circuits such that if $x \in A_{y e s}$ then $V_{x}$ accepts with probability at least $1-2^{-r}$, and if $x \in A_{n o}$ then $V_{x}$ accepts with probability at most $2^{-r}$, where $r$ is any polynomial. More precisely, let $V_{x}$ be the polynomial-size quantum circuit, which acts on $n=$ poly $(|x|)$ qubits, corresponding to the instance $x$. If we write $V_{x}\left|0^{n}\right\rangle$ as

$$
V_{x}\left|0^{n}\right\rangle=\sqrt{\alpha}|0\rangle \otimes\left|\phi_{0}\right\rangle+\sqrt{1-\alpha}|1\rangle \otimes\left|\phi_{1}\right\rangle
$$

with certain $(n-1)$-qubit states $\left|\phi_{0}\right\rangle$ and $\left|\phi_{1}\right\rangle$, we have $\alpha \geq 1-2^{-r}$ when $x \in A_{\text {yes }}$, and $\alpha \leq 2^{-r}$ when $x \in A_{n o}$. Let us consider the circuit of Fig. 3. We call it $U_{1}$. We also define

$$
U_{2} \equiv H^{\otimes n+m+1}
$$

We now show that deciding $x \in A_{\text {yes }}$ or $x \in A_{n o}$ can be reduced to a PDD-Max problem with $U_{1}$ and $U_{2}$, which means that PDD-Max is BQP-hard. In fact, note that

$$
U_{1}\left|0^{n+m+1}\right\rangle=\sqrt{\alpha}|0\rangle \otimes\left|0^{m}\right\rangle \otimes V_{x}^{\dagger}\left(|0\rangle \otimes\left|\phi_{0}\right\rangle\right)+\sqrt{1-\alpha}|1\rangle \otimes|+\rangle^{\otimes m} \otimes V_{x}^{\dagger}\left(|1\rangle \otimes\left|\phi_{1}\right\rangle\right) .
$$


Let $p_{z} \equiv\left|\left\langle z\left|U_{1}\right| 0^{n+m+1}\right\rangle\right|^{2}$ be the probability that we obtain $z \in\{0,1\}^{n+m+1}$ when we measure all qubits of $U_{1}\left|0^{n+m+1}\right\rangle$ in the computational basis. When $x \in A_{\text {yes }}$,

$$
p_{0^{n+m+1}}=\left|\left\langle 0^{n+m+1}\left|U_{1}\right| 0^{n+m+1}\right\rangle\right|^{2}=\alpha \mid\left\langle 0^{n}\left|V_{x}^{\dagger}\left(|0\rangle \otimes\left|\phi_{0}\right\rangle\right)\right|^{2}=\alpha^{2} \geq\left(1-2^{-r}\right)^{2} .\right.
$$

When $x \in A_{n o}$,

$$
\begin{aligned}
& p_{0 y}=\left|\left\langle 0 y\left|U_{1}\right| 0^{n+m+1}\right\rangle\right|^{2}=\alpha \mid\left\langle y\left|\left[\left|0^{m}\right\rangle \otimes V_{x}^{\dagger}\left(|0\rangle \otimes\left|\phi_{0}\right\rangle\right)\right]\right|^{2} \leq \alpha \leq 2^{-r}\right. \\
& p_{1 y}=\left|\left\langle 1 y\left|U_{1}\right| 0^{n+m+1}\right\rangle\right|^{2}=2^{-m}(1-\alpha) \mid\left\langle y_{m+1}, \ldots, y_{n+m}\left|V_{x}^{\dagger}\left(|1\rangle \otimes\left|\phi_{1}\right\rangle\right)\right|^{2} \leq 2^{-m}\right.
\end{aligned}
$$

for any $y \in\{0,1\}^{n+m}$. Let $q_{z} \equiv\left|\left\langle z\left|U_{2}\right| 0^{n+m+1}\right\rangle\right|^{2}$, where $z \in\{0,1\}^{n+m+1}$. Since $U_{2}=$ $H^{\otimes n+m+1}$, it is obvious that $q_{z}=\frac{1}{2^{n+m+1}}$ for any $z$. Therefore, when $x \in A_{\text {yes }}$,

$$
\left|p_{0^{n+m+1}}-q_{0^{n+m+1}}\right|=\left|p_{0^{n+m+1}}-\frac{1}{2^{n+m+1}}\right| \geq\left(1-2^{-r}\right)^{2}-2^{-(n+m+1)},
$$

and when $x \in A_{n o}$,

$$
\begin{aligned}
\left|p_{z}-q_{z}\right| & =\left|p_{z}-\frac{1}{2^{n+m+1}}\right| \\
& \leq p_{z}+\frac{1}{2^{n+m+1}} \\
& \leq \max \left(2^{-m}, 2^{-r}\right)+2^{-(n+m+1)}
\end{aligned}
$$

for any $z \in\{0,1\}^{n+m+1}$. In this way, we have shown that PDD-Max is BQP-hard.

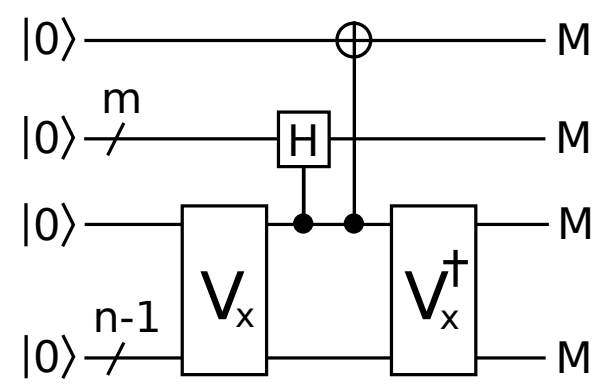

FIG. 3: The circuit $U_{1}$ used to show the BQP-hardness of PDD-Max.

\section{B. In BQP}

We next show that PDD-Max is in BQP. We show that the following BQP algorithm solves PDD-Max with a $1 / \operatorname{poly}(N)$ completeness-soundness gap (i.e., the gap of acceptance probabilities between the yes-instances and the no-instances is lowerbounded by $1 / \operatorname{pol} y(N)$ ): 
1. Flip a fair coin $s \in\{0,1\}$. Generate $U_{s+1}\left|0^{N}\right\rangle$, and measure each qubit in the computational basis. Let $z \in\{0,1\}^{N}$ be the measurement result.

2. Repeat the following for $i=1,2, \ldots, T$, where $T$ is a polynomial of $N$ specified later.

2-a. Generate $U_{1}\left|0^{N}\right\rangle$, and measure all qubits in the computational basis. If the result is $z$, then set $X_{i}=1$. Otherwise, set $X_{i}=0$.

3. Calculate

$$
\tilde{p}_{z} \equiv \frac{1}{T} \sum_{i=1}^{T} X_{i}
$$

Note that $E\left(X_{i}\right)=1 \times p_{z}+0 \times\left(1-p_{z}\right)=p_{z}$, where $p_{z} \equiv\left|\left\langle z\left|U_{1}\right| 0^{N}\right\rangle\right|^{2}$. Therefore, from the Chernoff-Hoeffding bound, $\left|\tilde{p}_{z}-p_{z}\right| \leq \epsilon$ with probability larger than $1-2 e^{-\frac{T \epsilon^{2}}{2}}$. In a similar way, calculate the estimator $\tilde{q}_{z}$ of $q_{z} \equiv\left|\left\langle z\left|U_{2}\right| 0^{N}\right\rangle\right|^{2}$. From the ChernoffHoeffding bound, $\left|\tilde{q}_{z}-q_{z}\right| \leq \epsilon$ with probability larger than $1-2 e^{-\frac{T \epsilon^{2}}{2}}$.

4. Calculate $\left|\tilde{p}_{z}-\tilde{q}_{z}\right|$. If $\left|\tilde{p}_{z}-\tilde{q}_{z}\right| \geq a-\frac{a-b}{4}$, accept. Otherwise, reject.

The intuitive idea of this algorithm is as follows. By the definition of the PDD-Max, if the answer of the PDD-Max is yes, there exists $z$ such that $\left|p_{z}-q_{z}\right|$ is large. In this case, the probability of obtaining such $z$ in step 1 is large. Therefore let us assume that we obtain such $z$ in step 1 . The step 1 is, in other words, the process to find a candidate of the solution. In steps 2 and 3, probabilities $p_{z}$ and $q_{z}$ are estimated by using the Chernoff-Hoeffding bound. Finally, in step 4, we check whether $\left|p_{z}-q_{z}\right|$ is indeed large, and accept with high probability since it is actually large. If the answer of the PDD-Max is no, on the other hand, there is no $z$ such that $\left|p_{z}-q_{z}\right|$ is large, and therefore in step 4 we do not conclude that $\left|p_{z}-q_{z}\right|$ is large except for some failure probability. (Note that for general $U_{1}$ and $U_{2}$, estimating $p_{z}$ and $q_{z}$ seems to require BQP power, since it seems to be necessary to generate $U_{1}\left|0^{N}\right\rangle$ and $U_{2}\left|0^{N}\right\rangle$. We will see in the next section that if $U_{1}$ and $U_{2}$ are restricted in $\mathrm{FH}_{2}$, the estimation of $p_{z}$ and $q_{z}$ can be done in classical polynomial time, and therefore PDD-Max has a Merlin-Arthur system with quantum polynomial-time Merlin.)

Now let us give a more precise proof that PDD-Max is in BQP. First, let us consider the case when the answer to PDD-Max is YES. If $z$ obtained in step 1 satisfies $\left|p_{z}-q_{z}\right| \geq a$, and if $\tilde{p}_{z}$ and $\tilde{q}_{z}$ calculated in steps 2 and 3 satisfy $\left|\tilde{p}_{z}-p_{z}\right| \leq \frac{a-b}{8}$ and $\left|\tilde{q}_{z}-q_{z}\right| \leq \frac{a-b}{8}$, we 
definitely accept in step 4, because

$$
\begin{aligned}
a & \leq\left|p_{z}-q_{z}\right| \\
& \leq\left|p_{z}-\tilde{p}_{z}\right|+\left|\tilde{p}_{z}-q_{z}\right| \\
& \leq\left|p_{z}-\tilde{p}_{z}\right|+\left|\tilde{p}_{z}-\tilde{q}_{z}\right|+\left|\tilde{q}_{z}-q_{z}\right| \\
& \leq\left|\tilde{p}_{z}-\tilde{q}_{z}\right|+\frac{a-b}{4},
\end{aligned}
$$

and therefore

$$
\left|\tilde{p}_{z}-\tilde{q}_{z}\right| \geq a-\frac{a-b}{4}
$$

The probability $\eta$ of occurring such an event is calculated to be

$$
\begin{aligned}
\eta \equiv & \operatorname{Pr}\left[z \text { obtained in step } 1 \text { satisfies }\left|p_{z}-q_{z}\right| \geq a\right] \\
& \times \operatorname{Pr}\left[\left|\tilde{p}_{z}-p_{z}\right| \leq \frac{a-b}{8}\right] \times \operatorname{Pr}\left[\left|\tilde{q}_{z}-q_{z}\right| \leq \frac{a-b}{8}\right] \\
= & \left(\sum_{z:\left|p_{z}-q_{z}\right| \geq a} \frac{p_{z}+q_{z}}{2}\right) \times \operatorname{Pr}\left[\left|\tilde{p}_{z}-p_{z}\right| \leq \frac{a-b}{8}\right] \times \operatorname{Pr}\left[\left|\tilde{q}_{z}-q_{z}\right| \leq \frac{a-b}{8}\right] \\
\geq & \frac{a}{2}\left(1-2 e^{-\frac{T(a-b)^{2}}{128}}\right)^{2} \\
\geq & \frac{a}{2}\left(1-2 e^{-k}\right)^{2},
\end{aligned}
$$

where we have taken $T \geq \frac{128 k}{(a-b)^{2}}$ and $k$ is any polynomial of $N$. Therefore, the acceptance probability $p_{a c c}$ of our protocol is lowerbounded as

$$
p_{a c c} \geq \eta \geq \frac{a}{2}\left(1-2 e^{-k}\right)^{2} \equiv \alpha
$$

Next, let us consider the case when the answer to PDD-Max is NO. If $\left|\tilde{p}_{z}-p_{z}\right| \leq \frac{a-b}{8}$ and $\left|\tilde{q}_{z}-q_{z}\right| \leq \frac{a-b}{8}$, which occurs with probability $\geq\left(1-2 e^{-k}\right)^{2}$, we definitely reject, because

$$
\begin{aligned}
\left|\tilde{p}_{z}-\tilde{q}_{z}\right| & \leq\left|\tilde{p}_{z}-p_{z}\right|+\left|p_{z}-\tilde{q}_{z}\right| \\
& \leq\left|\tilde{p}_{z}-p_{z}\right|+\left|p_{z}-q_{z}\right|+\left|q_{z}-\tilde{q}_{z}\right| \\
& \leq \frac{a-b}{4}+b \\
& <a-\frac{a-b}{4} .
\end{aligned}
$$

The rejection probability $p_{r e j}$ is therefore lowerbounded as

$$
p_{r e j} \geq\left(1-2 e^{-k}\right)^{2}
$$


Therefore, the acceptance probability $p_{a c c}$ is upperbounded as

$$
p_{a c c}=1-p_{r e j} \leq 1-\left(1-2 e^{-k}\right)^{2}=4 e^{-k}-4 e^{-2 k} \equiv \beta \text {. }
$$

The completeness-soundness gap is therefore

$$
\alpha-\beta=\frac{a}{2}\left(1-2 e^{-k}\right)^{2}-\left(4 e^{-k}-4 e^{-2 k}\right) \geq \frac{1}{\operatorname{poly}(N)}
$$

for sufficiently large $k$, which shows that PDD-Max is in BQP.

\section{PROOF OF THEOREM 13}

In this section, we show Theorem 13. Before giving the proof, let us explain the intuitive idea. In Sec. $\mathrm{VB}$, we have seen that PDD-Max is in BQP for general $U_{1}$ and $U_{2}$. In step 1 of the algorithm, a candidate of the solution, i.e., $z$ such that $\left|p_{z}-q_{z}\right| \geq a$, is obtained by doing quantum computing. In step 2, another quantum computing is necessary to estimate $p_{z}$ and $q_{z}$. In the following we will see that if $U_{1}$ and $U_{2}$ are the HC1Q model, estimations of $p_{z}$ and $q_{z}$ can be done in classical polynomial-time. Therefore, we can construct the following Merlin-Arthur protocol with quantum polynomial-time Merlin:

1. Merlin does the step 1 of the protocol in Sec. VB, i.e., Merlin generates $z$ and sends it to Arthur.

2. Arthur verifies $\left|p_{z}-q_{z}\right|$ is large.

Now let us show Theorem 13. Our Merlin-Arthur protocol with quantum polynomial-time Merlin runs as follows.

1. If Merlin is honest, he flips a fair coin $s \in\{0,1\}$. He next generates $U_{s+1}\left|0^{N}\right\rangle$, and measures each qubit in the computational basis to obtain the result $z \in\{0,1\}^{N}$. He sends $z$ to Arthur. If Merlin is malicious, his computational ability is unbounded, and what he sends to Arthur can be any $N$ bit string.

2. Let $T$ be a polynomial of $N$ specified later. Arthur generates $\left\{x^{i}\right\}_{i=1}^{T}$ and $\left\{y^{i}\right\}_{i=1}^{T}$, where each of $x^{i} \in\{0,1\}^{N-1}$ and $y^{i} \in\{0,1\}^{N-1}$ is a uniformly and independently chosen random $N-1$ bit string. 
3. Arthur calculates

$$
\tilde{p}_{z} \equiv \frac{1}{T} \sum_{i=1}^{T} f\left(x^{i}, y^{i}\right)
$$

where

$$
f(x, y) \equiv(-1)^{\sum_{j=1}^{N-1}\left[C_{j}(x 0) \cdot z_{j}+C_{j}(y 0) \cdot z_{j}\right]} \delta_{C_{N}(x 0), z_{N}} \delta_{C_{N}(y 0), z_{N}} .
$$

Here,

$$
C:\{0,1\}^{N} \ni w \mapsto C(w) \in\{0,1\}^{N}
$$

is the classical circuit in $U_{1}$,

$$
x 0 \equiv\left(x_{1}, \ldots, x_{N-1}, 0\right),
$$

$C_{j}(x 0)$ is the $j$ th bit of $C(x 0) \in\{0,1\}^{N}$, and $z_{j}$ is the $j$ th bit of $z \in\{0,1\}^{N}$. We call $\tilde{p}_{z}$ the estimator of $p_{z}$. Note that $\tilde{p}_{z}$ can be calculated in classical poly $(N)$ time.

4. In a similar way, Arthur calculates the estimator $\tilde{q}_{z}$ of $q_{z}$. If

$$
\left|\tilde{p}_{z}-\tilde{q}_{z}\right| \geq a-\frac{a-b}{4},
$$

Arthur accepts. Otherwise, he rejects.

Note that

$$
p_{z} \equiv\left|\left\langle z\left|U_{1}\right| 0^{N}\right\rangle\right|^{2}=\frac{1}{2^{2(N-1)}} \sum_{x, y \in\{0,1\}^{N-1}} f(x, y) .
$$

Its derivation is given in Appendix C, If we set $X_{i}=f\left(x^{i}, y^{i}\right)$, we have

$$
E\left(X_{i}\right)=\frac{1}{2^{2(N-1)}} \sum_{x, y \in\{0,1\}^{N-1}} f(x, y)=p_{z} .
$$

Therefore, from the Chernoff-Hoeffding bound, the estimator $\tilde{p}_{z}$ satisfies $\left|\tilde{p}_{z}-p_{z}\right| \leq \epsilon$ with probability $\geq 1-2 e^{-\frac{T \epsilon^{2}}{2}}$. For $\tilde{q}_{z}$, a similar result holds. In the above protocol, the honest prover is enough to be quantum polynomial-time.

Now we show that the above protocol can verify PDD-Max. The proof is similar to that for the BQP case given in Sec. V First, let us consider the case when the answer to PDDMax is yes. If the $z$ that Merlin sends to Arthur satisfies $\left|p_{z}-q_{z}\right| \geq a$, and the estimators 
$\tilde{p}_{z}$ and $\tilde{q}_{z}$ that Arthur calculates satisfy $\left|\tilde{p}_{z}-p_{z}\right| \leq \frac{a-b}{8}$ and $\left|\tilde{q}_{z}-q_{z}\right| \leq \frac{a-b}{8}$, Arthur definitely accepts, because

$$
\begin{aligned}
\left|\tilde{p}_{z}-\tilde{q}_{z}\right| & \geq\left|p_{z}-q_{z}\right|-\left|\tilde{p}_{z}-p_{z}\right|-\left|\tilde{q}_{z}-q_{z}\right| \\
& \geq a-\frac{a-b}{4} .
\end{aligned}
$$

Taking the honest prover in step 1, the probability $\eta$ of occurring such an event is calculated to be

$$
\begin{aligned}
\eta \equiv & \operatorname{Pr}\left[\text { Merlin obtains } z \text { such that }\left|p_{z}-q_{z}\right| \geq a\right] \\
& \times \operatorname{Pr}\left[\left|\tilde{p}_{z}-p_{z}\right| \leq \frac{a-b}{8}\right] \times \operatorname{Pr}\left[\left|\tilde{q}_{z}-q_{z}\right| \leq \frac{a-b}{8}\right] \\
= & \left(\sum_{z:\left|p_{z}-q_{z}\right| \geq a} \frac{p_{z}+q_{z}}{2}\right) \times \operatorname{Pr}\left[\left|\tilde{p}_{z}-p_{z}\right| \leq \frac{a-b}{8}\right] \times \operatorname{Pr}\left[\left|\tilde{q}_{z}-q_{z}\right| \leq \frac{a-b}{8}\right] \\
\geq & \frac{a}{2}\left(1-2 e^{-\frac{T(a-b)^{2}}{128}}\right)^{2} \geq \frac{a}{2}\left(1-2 e^{-k}\right)^{2}
\end{aligned}
$$

where we have taken $T \geq \frac{128 k}{(a-b)^{2}}$ and $k$ is any polynomial of $N$. Therefore, the probability $p_{\text {acc }}$ that Arthur accepts in our protocol is lowerbounded as

$$
p_{a c c} \geq \frac{a}{2}\left(1-2 e^{-k}\right)^{2} \equiv \alpha
$$

Next, let us consider the case when the answer to PDD-Max is no. If $\left|\tilde{p}_{z}-p_{z}\right| \leq \frac{a-b}{8}$ and $\left|\tilde{q}_{z}-q_{z}\right| \leq \frac{a-b}{8}$, which occurs with probability $\geq\left(1-2 e^{-k}\right)^{2}$, Arthur definitely rejects, because

$$
\begin{aligned}
\left|\tilde{p}_{z}-\tilde{q}_{z}\right| & \leq\left|\tilde{p}_{z}-p_{z}\right|+\left|p_{z}-q_{z}\right|+\left|q_{z}-\tilde{q}_{z}\right| \\
& \leq b+\frac{a-b}{4}<a-\frac{a-b}{4} .
\end{aligned}
$$

Therefore, the probability $p_{a c c}$ that Arthur accepts in our protocol is upperbounded as

$$
p_{a c c} \leq 1-\left(1-2 e^{-k}\right)^{2}=4 e^{-k}-4 e^{-2 k} \equiv \beta .
$$

The completeness-soundness gap is therefore

$$
\alpha-\beta=\frac{a}{2}\left(1-2 e^{-k}\right)^{2}-\left(4 e^{-k}-4 e^{-2 k}\right) \geq \frac{1}{\operatorname{poly}(N)}
$$

for sufficiently large $k$, which shows that PDD-Max has a Merlin-Arthur system with quantum polynomial-time Merlin. 
Remarks. To conclude this section, we provide some remarks. Note that the fact that $p_{z}$ and $q_{z}$ can be estimated in classical polynomial-time seems to be a special property only for some circuits (such as those in $\mathrm{FH}_{2}$ ), and we do not know how to do that for other general circuits. (In particular, in Sec. VIIB, we will explain why the technique we use for $\mathrm{FH}_{2}$ circuits will not work for other circuits, such as $\mathrm{FH}_{3}$ circuits.) Furthermore, note that what Arthur can do is to estimate $p_{z}$ within an additive error given $z$ : he cannot sample $\left\{p_{z}\right\}_{z}$. (If he can do it, he can solve factoring and Simon's problem, for example.)

\section{DISCUSSION}

\section{A. Generalizations to other circuits}

In the proof of Theorem 13 (Sec. VI), we have considered the HC1Q model. Similar proofs hold for other circuits in $\mathrm{FH}_{2}$. For example, let us consider the IQP model. For the IQP model, $f(x, y)$ is a complex-valued function, but we can use the Chernoff-Hoeffding bound for complex random variables introduced in Ref. [38].

The other example is the $\mathrm{HCm} \mathrm{Q}$ model. We can show a similar result for it. In fact, the probability $p_{s, t}$ of obtaining the result $(s, t) \in\{0,1\}^{N-m} \times\{0,1\}^{m}$ is

$p_{s, t}=\frac{1}{2^{2(N-m)}} \sum_{x, y \in\{0,1\}^{N-m}}(-1)^{C_{1, \ldots, N-m}\left(x 0^{m}\right) \cdot s+C_{1, \ldots, N-m}\left(y 0^{m}\right) \cdot s} \delta_{t, C_{N-m+1, \ldots, N}\left(x 0^{m}\right)} \delta_{t, C_{N-m+1, \ldots, N}\left(y 0^{m}\right)}$,

and therefore the Chernoff-Hoeffding bound argument can be used. Here, $C_{1, \ldots, N-m}\left(x 0^{m}\right)$ is the first $N-m$ bits of $C\left(x 0^{m}\right)$, and $C_{N-m+1, \ldots, N}\left(x 0^{m}\right)$ is the last $m$ bits of $C\left(x 0^{m}\right)$.

Furthermore, we would like to point out that PDD-Max can has a Merlin-Arthur system with quantum polynomial-time Merlin for other circuits outside of $\mathrm{FH}_{2}$. The essential point of our proof is that the output probability distributions, $p_{z}$ and $q_{z}$, of $U_{1}$ and $U_{2}$ can be classically efficiently estimated. This property itself is not restricted to circuits in $\mathrm{FH}_{2}$, and therefore we believe that our results should hold for many other circuits outside of $\mathrm{FH}_{2}$.

\section{B. $\mathbf{F H}_{3}$}

$\mathrm{FH}_{2}$ circuits have nice structures such that $p_{z}$ and $q_{z}$ can be estimated in classical polynomial-time. We do not know how to do the same thing for other circuits such as 
those in $\mathrm{FH}_{3}$. The reason is as follows. From a similar calculation given in Appendix $\mathrm{C}$, we can show that the probability $p_{z} \equiv\left|\left\langle z|U| 0^{N}\right\rangle\right|^{2}$ for an $\mathrm{FH}_{3}$ circuit $U$ satisfies

$$
p_{z}=\frac{1}{2^{M}} \sum_{x \in\{0,1\}^{M}} f(x)
$$

for certain $M=\operatorname{poly}(N)$ and a certain function $f$. However, in this case, we no longer have $|f(x)| \leq 1$, but $f$ is exponentially increasing as a function of $N: f(x)=2^{s(N)} g(x)$, where $|g(x)| \leq 1$ and $s$ is a polynomial. Then, by using the Chernoff-Hoeffding bound, we can obtain an estimator $\xi$ that satisfies

$$
\left|\xi-\frac{1}{2^{M}} \sum_{x} g(x)\right| \leq \epsilon
$$

with probability larger than $1-2 e^{-\frac{T \epsilon^{2}}{2}}$. The above inequality means

$$
\left|\xi-\frac{p_{z}}{2^{s(N)}}\right| \leq \epsilon
$$

which also means

$$
\left|2^{s(N)} \xi-p_{z}\right| \leq 2^{s(N)} \epsilon
$$

In order to make $2^{s(N)} \epsilon=O(1 /$ poly $(N)), \epsilon$ must be exponentially small, which means that $T$ must be exponentially large. Therefore, we cannot obtain a $1 /$ poly $(N)$-precision estimator of $p_{z}$ in classical polynomial time. Note that it is known that as long as we use $f$ as a black box, the Chernoff-Hoeffding type bounds are optimal (up to some factors) [39]. This is the reason why our previous proof does not work for other circuits.

\section{Acknowledgments}

We thank Keisuke Fujii, Seiichiro Tani, and Francois Le Gall for discussion. TM is supported by JST ACT-I No.JPMJPR16UP, JST PRESTO, and the Grant-in-Aid for Young Scientists (B) No.JP17K12637 of JSPS. YT is supported by the Program for Leading Graduate Schools: Interactive Materials Science Cadet Program and JSPS Grant-in-Aid for JSPS Research Fellow No.JP17J03503. HN is supported by the Grant-in-Aid for Scientific Research (A) Nos.26247016, 16H01705 and (C) No.16K00015 of JSPS. 


\section{Appendix A: Example}

Let us consider the simple example where $n=2, t=1$, and $U=u_{1}=X \otimes I$. In this case, $U^{\prime}=u_{3} u_{2} u_{1}$, where

$$
\begin{aligned}
& u_{2}=H \otimes I, \\
& u_{3}=I \otimes H .
\end{aligned}
$$

The computational tree that represents the non-deterministic algorithm corresponding to $U^{\prime}$ is given in Fig. 4. Each Hadamard gate in $U^{\prime}$ corresponds to a non-deterministic transition. The bit string $z$ represents a basis state, and the bit $s$ represents the sign of a basis state. If we superpose $(-1)^{s(y)}|z(y)\rangle$ for all computational path $y \in\{0,1\}^{2}$, we obtain

$$
\sum_{y \in\{0,1\}^{2}}(-1)^{s(y)}|z(y)\rangle=\left[(-1)^{0}|00\rangle+(-1)^{0}|01\rangle+(-1)^{1}|10\rangle+(-1)^{1}|11\rangle\right]=\sqrt{2^{2}} U^{\prime}\left|0^{2}\right\rangle .
$$

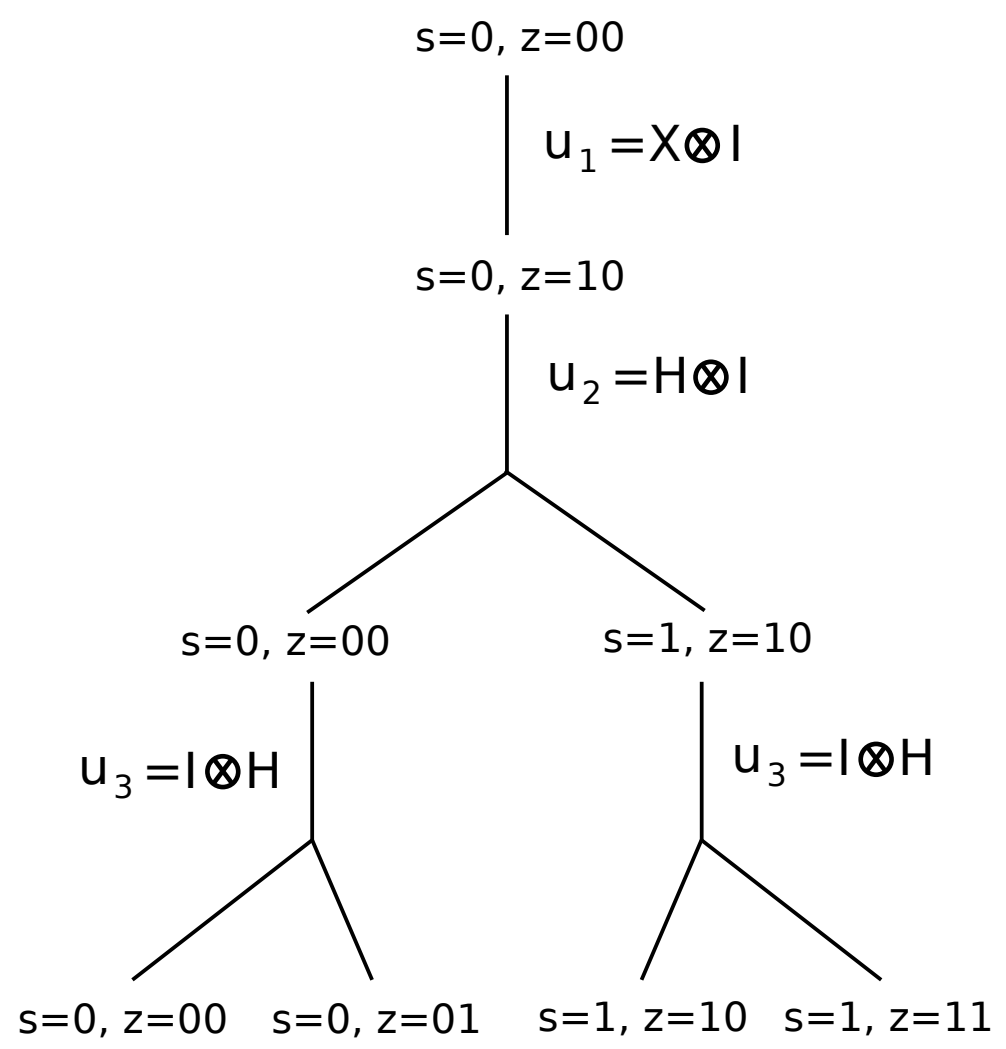

FIG. 4: The computational tree of the non-deterministic algorithm. 


\section{Appendix B: Derivation of Eq. (3)}

In this appendix, we show Eq. (3). The initial state is $\left|0^{N}\right\rangle$. We first apply $H^{\otimes(N-1)} \otimes I$. Then the state becomes

$$
\frac{1}{\sqrt{2^{N-1}}} \sum_{x \in\{0,1\}^{N-1}}|x\rangle|0\rangle
$$

We apply the classical gate $C$ to obtain

$$
\frac{1}{\sqrt{2^{N-1}}} \sum_{x \in\{0,1\}^{N-1}}|C(x 0)\rangle .
$$

If we project this state onto $\left(H^{\otimes k}|z\rangle\langle z| H^{\otimes k}\right) \otimes I^{\otimes N-k}$, where $z \in\{0,1\}^{k}$, the state becomes

$$
\frac{1}{\sqrt{2^{N-1}}} \frac{1}{\sqrt{2^{k}}} \sum_{x \in\{0,1\}^{N-1}}(-1)^{C_{1, \ldots, k}(x 0) \cdot z}\left(H^{\otimes k}|z\rangle\right) \otimes\left|C_{k+1, \ldots, N}(x 0)\right\rangle .
$$

If we calculate its norm, which is the probability $p_{z}$, we obtain Eq. (3).

\section{Appendix C: Derivation of Eq. (4)}

In this appendix, we show Eq. (4). The initial state is $\left|0^{N}\right\rangle$. We first apply $H^{\otimes(N-1)} \otimes I$. Then the state becomes

$$
\frac{1}{\sqrt{2^{N-1}}} \sum_{x \in\{0,1\}^{N-1}}|x\rangle|0\rangle .
$$

We apply the classical gate $C$ to obtain

$$
\frac{1}{\sqrt{2^{N-1}}} \sum_{x \in\{0,1\}^{N-1}}|C(x 0)\rangle .
$$

We apply the final Hadamard $H^{\otimes(N-1)} \otimes I$ to obtain

$$
\frac{1}{2^{N-1}} \sum_{x, y \in\{0,1\}^{N-1}}(-1)^{\sum_{j=1}^{N-1} C_{j}(x 0) \cdot y_{j}}|y\rangle\left|C_{N}(x 0)\right\rangle=U_{1}\left|0^{N}\right\rangle,
$$

where $C_{j}(x 0)$ is the $j$ th bit of $C(x 0)$ and $y_{j}$ is the $j$ th bit of $y$. Therefore

$$
\left\langle z\left|U_{1}\right| 0^{N}\right\rangle=\frac{1}{2^{N-1}} \sum_{x \in\{0,1\}^{N-1}}(-1)^{\sum_{j=1}^{N-1} C_{j}(x 0) \cdot z_{j}} \delta_{C_{N}(x 0), z_{N}}
$$


which finally gives Eq. (4)

$$
p_{z}=\left|\left\langle z\left|U_{1}\right| 0^{N}\right\rangle\right|^{2}=\frac{1}{2^{2(N-1)}} \sum_{x, y \in\{0,1\}^{N-1}} f(x, y) .
$$

[1] Y. Shi, Quantum and classical tradeoffs. Theoretical Computer Science 344, 335 (2005). DOI:10.1016/j.tcs.2005.03.053

[2] D. R. Simon, On the power of quantum computation. Proceedings of the 35th Annual Symposium on Foundations of Computer Science (FOCS 1994), p.116 (1994). DOI:10.1137/S0097539796298637

[3] P. Shor, Algorithms for quantum computation: discrete logarithms and factoring. Proceedings of the 35th Annual Symposium on Foundations of Computer Science (FOCS 1994), p.124 (1994). DOI:10.1109/SFCS.1994.365700

[4] M. J. Bremner, R. Jozsa, and D. J. Shepherd, Classical simulation of commuting quantum computations implies collapse of the polynomial hierarchy. Proc. R. Soc. A 467, 459 (2011). DOI:10.1098/rspa.2010.0301

[5] M. J. Bremner, A. Montanaro, and D. J. Shepherd, Average-case complexity versus approximate simulation of commuting quantum computations. Phys. Rev. Lett. 117, 080501 (2016). DOI:10.1103/PhysRevLett.117.080501

[6] B. M. Terhal and D. P. DiVincenzo, Adaptive quantum computation, constant depth quantum circuits and Arthur-Merlin games. Quant. Inf. Comput. 4, 134 (2004). DOI:10.26421/QIC4.2

[7] S. Aaronson and A. Arkhipov, The computational complexity of linear optics. Theory of Computing 9, 143 (2013). DOI:10.1145/1993636.1993682

[8] E. Knill, and R. Laflamme, Power of one bit of quantum information. Phys. Rev. Lett. 81, 5672 (1998). DOI:10.1103/PhysRevLett.81.5672

[9] T. Morimae, K. Fujii, and J. F. Fitzsimons, Hardness of classically simulating the one clean qubit model. Phys. Rev. Lett. 112, 130502 (2014). DOI:10.1103/PhysRevLett.112.130502

[10] T. Morimae, Hardness of classically sampling one clean qubit model with constant total variation distance error. Phys. Rev. A 96, 040302(R) (2017). DOI:10.1103/PhysRevA.96.040302

[11] K. Fujii, H. Kobayashi, T. Morimae, H. Nishimura, S. Tamate, and S. Tani, Impossibility of classically simulating one-clean-qubit computation. Phys. Rev. Lett. 120, 200502 (2018). 
DOI:10.1103/PhysRevLett.120.200502

[12] K. Fujii, H. Kobayashi, T. Morimae, H. Nishimura, S. Tamate, and S. Tani, Power of quantum computation with few clean qubits. Proceedings of 43rd International Colloquium on Automata, Languages, and Programming (ICALP 2016), p.13:1. DOI:10.4230/LIPIcs.ICALP.2016.13

[13] B. Fefferman and C. Umans, The power of quantum Fourier sampling. arXiv:1507.05592

[14] A. Bouland, J. F. Fitzsimons, and D. E. Koh, Quantum advantage from conjugated Clifford circuits. Proceedings of the 33rd Computational Complexity Conference (CCC2018). DOI:10.4230/LIPIcs.CCC.2018.21

[15] A. Bouland, B. Fefferman, C. Nirkhe, and U. Vazirani, On the complexity and verification of quantum random circuit sampling. Nat. Phys. 2018 DOI:10.1038/s41567-018-0318-2

[16] D. Aharonov and U. Vazirani, Is quantum mechanics falsifiable? A computational perspective on the foundations of quantum mechanics. arXiv:1206.3686

[17] T. Morimae, D. Nagaj, and N. Schuch, Quantum proofs can be verified using only single-qubit measurements. Phys. Rev. A 93, 022326 (2016). DOI:10.1103/PhysRevA.93.022326

[18] J. F. Fitzsimons, M. Hajdušek, and T. Morimae, Post hoc verification of quantum computation. Phys. Rev. Lett. 120, 040501 (2018). DOI:10.1103/PhysRevLett.120.040501

[19] J. F. Fitzsimons and E. Kashefi, Unconditionally verifiable blind computation. Phys. Rev. A 96, 012303 (2017). DOI:10.1103/PhysRevA.96.012303

[20] D. Aharonov, M. Ben-Or, E. Eban, and U. Mahadev, Interactive proofs for quantum computations. arXiv:1704.04487

[21] M. Hayashi and T. Morimae, Verifiable measurement-only blind quantum computing with stabilizer testing. Phys. Rev. Lett. 115, 220502 (2015). DOI:10.1103/PhysRevLett.115.220502

[22] A. Broadbent, How to verify quantum computation. Theory of Computing 14, 1 (2018). DOI:10.4086/toc.2018.v014a011

[23] A. Gheorghiu, T. Kapourniotis, and E. Kashefi, Verification of quantum computation: an overview of existing approaches. arXiv:1709.06984

[24] M. McKague, Interactive proofs for BQP via self-tested graph states. Theory of Computing 12, 1 (2016). DOI:10.4086/toc.2016.v012a003

[25] Z. Ji, Classical verification of quantum proofs. Proceedings of the 48th annual ACM symposium on Theory of Computing (STOC 2016) p.885 (2016). DOI:10.1145/2897518.2897634 
[26] B. W. Reichardt, F. Unger, and U. Vazirani, Classical command of quantum systems. Nature 496, 456 (2013). DOI:10.1038/nature12035

[27] IP is the class of promise problems verified by an interactive proof system with a BPP verifier and a prover with unlimited computational ability.

[28] D. Aharonov and A. Green, A quantum inspired proof of $\mathrm{P} \# \mathrm{P} \subseteq \mathrm{IP}$. arXiv:1710.09078

[29] U. Mahadev, Classical verification of quantum computations. arXiv:1804.01082

[30] E. Bernstein and U. Vazirani, Quantum complexity theory. SIAM Journal on Computing 26, 1411 (1997). DOI:10.1137/S0097539796300921

[31] M. McKague, Interactive proofs with efficient quantum prover for recursive Fourier sampling. Chicago Journal of Theoretical Computer Science 6, 1 (2012). DOI:10.4086/cjtcs.0006

[32] T. F. Demarie, Y. Ouyang, and J. F. Fitzsimons, Classical verification of quantum circuits containing few basis changes. Phys. Rev. A 97, 042319 (2018). DOI:10.1103/PhysRevA.97.042319

[33] F. Le Gall, T. Morimae, H. Nishimura, and Y. Takeuchi, Interactive proofs with polynomialtime quantum prover for computing the order of solvable groups. arXiv:1805.03385

[34] Merlin-Arthur (MA) is the class of promise problems that can be verified by a BPP verifier and a prover whose computational ability is unlimited. In MA, the prover first sends a bit string to the verifier, and the verifier then does the computing for the verification.

[35] Note that the $(n+1)$-qubit Toffoli gate in Fig. 2 can be implemented with a polynomially many Toffoli gates by using ancilla qubits [36]. It is known that ancilla qubits do not need to be initialized [36]. Therefore, we can use $|+\rangle$ states as ancilla qubits, which keeps the circuit of Fig. 2 in the form of Fig. 1(a).

[36] A. Barenco, C. H. Bennett, R. Cleve, D. P. DiVincenzo, N. Margolus, P. Shor, T. Sleator, J. A. Smolin, H. Weinfurter, Elementary gates for quantum computation. Phys. Rev. A 523457 (1995). DOI:10.1103/PhysRevA.52.3457

[37] L. M. Adleman, J. DeMarrais, and M. A. Huang, Quantum Computability. SIAM J. Comput. 261524 (1997). DOI:10.1137/S0097539795293639

[38] M. Schwarz and M. Van den Nest, Simulating quantum circuits with sparse output distributions. arXiv:1310.6749

[39] R. Canetti, G. Even, and O. Goldreich, Lower bounds for sampling algorithms for estimating the average. Information Processing Letters 53, 17 (1995). 